\title{
Folded shift multiplexing
}

\author{
Christophe Moser and Wenhai Liu
}

Ondax, Inc., 850 East Duarte Road, Monrovia, California 91016

\section{Yeshaiahu Fainman}

\begin{abstract}
Department of Electrical and Computer Engineering, University of California, San Diego, San Diego, California 92093
\end{abstract}
Demetri Psaltis

Department of Electrical Engineering, California Institute of Technology, Pasadena, California 91125

Received December 5, 2002

\begin{abstract}
Shift multiplexing is a holographic recording method that uses a spherical reference wave. We extend the principle to a thin slab of holographic material that acts as a waveguide. Total internal reflection folds the reference spherical beam in one dimension. We demonstrate that the shift selectivity with the folded spherical beam is independent of the slab thickness but depends instead on the numerical aperture of the coupled spherical wave. A shift selectivity of $0.5 \mu \mathrm{m}$ has been achieved with a 1-mm-thick $\mathrm{LiNbO}_{3}$ crystal and 50 high-definition data pages are recorded with this method. () 2003 Optical Society of America

OCIS codes: $090.0090,210.0210,090.7330,090.4220$.
\end{abstract}

Holograms have been recorded in guided media such as fibers. ${ }^{1,2}$ Alternatively, the guiding medium itself (a multimode fiber) has been used for the generation of a random reference wave. ${ }^{3}$ The novel approach presented in this Letter uses a slab waveguide as both the reference-guiding medium and the holographic storage medium for multiplexing. Shift multiplexing $^{4,5}$ was proposed as a holographic storage method. Holograms are multiplexed by rotation of the holographic medium with respect to a fixed spherical reference beam and an object data beam. ${ }^{6-9}$ Figure 1 illustrates shift multiplexing in a $90^{\circ}$ geometry recording. We use this geometry to demonstrate folded shift multiplexing. Extension to different angles is straightforward. The shift selectivity $\delta$ for the $90^{\circ}$ geometry can be computed analytically ${ }^{5}$ and is given by

$$
\delta=\frac{\lambda z_{0}}{L}
$$

where $L$ is the crystal thickness, $z_{0}$ is the distance from the origin of the spherical wave to the center of the hologram, and $\lambda$ is the wavelength inside the medium. A thicker material yields a smaller shift selectivity according to Eq. (1) and therefore a larger storage density. However, a large material thickness is necessary to accommodate the diverging spherical beam. This is practically inconvenient because it creates bulky implementations, and some holographic materials (e.g., polymers ${ }^{10,11}$ ) cannot be made arbitrarily thick .

The idea of folded shift multiplexing is to replace the thick material with a thin slab as shown in Fig. 1(b). We show that the shift selectivity with the thin slab is equal to that of thick slabs. The low profile of the recording material permits storage architectures with a much smaller form factor while maintaining storage capacity.

In Fig. 1(b), the spherical wave is trapped in one dimension $(y)$ inside the crystal by total internal reflection. A cylindrical lens can be used to confine the beam in the $x$ direction. In this Letter we present the derivation of the shift selectivity with the slab waveguide and present experimental results to support the predictions.

We assume that the thickness $D$ of the slab is much larger than the wavelength of light, so the assumption of a continuum of modes is valid. Under this assumption, the wave inside the slab is still spherical but folded. Without losing generality, we consider only light trapping in the $y-z$ plane with linear TEpolarized light. A point $\mathrm{P}$ in the crystal slab [Fig. 1(b)] is positioned at the intersection of many rays, each having a different phase because of its

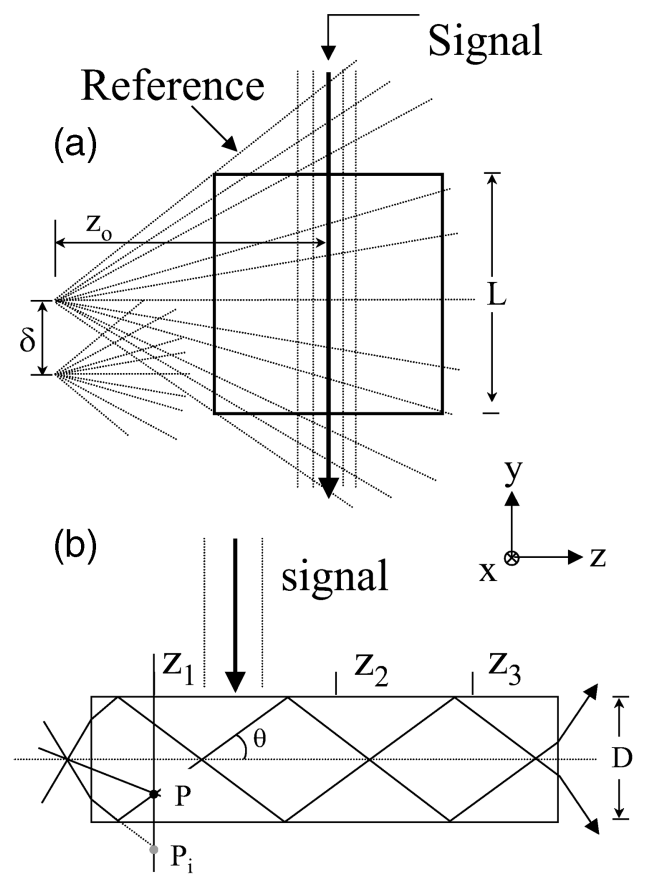

Fig. 1. (a) Shift multiplexing in the $90^{\circ}$ geometry. (b) Shift multiplexing by total internal reflection in a crystal slab. 
different path. Multiple rays intersecting at point $\mathrm{P}$ as a result of folding is equivalent to an unfolded wave extended beyond the crystal thickness. We must also take into account the phase shift $\phi$ caused by total internal reflection of the rays in the dielectric material. The phase shift $\phi$ of a ray reflected at the boundary is dependent on the angle of incidence $\theta$ as defined in Fig. 1(b) and given by ${ }^{12}$

$$
\tan \left(\frac{\phi}{2}\right)=\left(\frac{\sin ^{2} \theta_{c}}{\sin ^{2} \theta}-1\right)
$$

$\theta_{c}$ is the critical angle equal to a $\sin \left[1-\left(n_{1} / n_{2}\right)^{2}\right]^{1 / 2}$, where $n_{2}$ and $n_{1}$ are the indices inside and outside the slab, respectively.

We can write an expression for the folded spherical wave inside the slab waveguide by modifying the cylindrical wave front that we would normally obtain in free space by unfolding the wave inside the slab and introducing additional phase shifts to account for the total internal reflections. To calculate the proper wave-front modification, we can trace the rays originating at the focal plane of the cylindrical wave and diverging into the slab. Each time a ray hits the walls at an angle $\theta$, a phase given by Eq. (2) is added at that point in the wave front. In the paraxial approximation the total phase after multiple bounces is equal to

$$
\Phi(\theta)=\frac{z}{D / \theta} \phi(\theta) .
$$

Following this procedure with a Taylor expansion around $\phi=\pi / 2$ and neglecting third orders in $\theta=y / z$, we derive the folded cylindrical folded wave:

$$
\begin{aligned}
R(x, z)= & \frac{1}{j \lambda z} \exp \left(j 2 \pi \frac{z}{\lambda}\right) \\
& \times \exp \left(j \pi \frac{y^{2}}{\lambda z}\left[1-\frac{2 \lambda}{\pi D \sin \theta_{c}}\right]\right) \\
& \times \exp \left(j \pi \frac{y}{D}\right) .
\end{aligned}
$$

The wavelength $\lambda$ is much smaller than the thickness $D$ of the slab, so the term in brackets approaches unity and can therefore be neglected. The graphic representation of the wave front shown in Fig. 2 illustrates the small effect of the additional phase shift due to multiple reflections. Equation (4) is valid in the paraxial approximation. A practical value for the N.A. of the wave is given by the optics and is assumed to be $<0.65$; in this case the deviation from the paraxial approximation inside a material of index 2.2 is $<10 \%$. The derivation of the shift selectivity with this reference wave in the $90^{\circ}$ geometry can be readily computed from Ref. 5 and is given by

$$
\delta=\frac{\lambda}{2 \mathrm{~N} . \mathrm{A}},
$$

where N.A. is the numerical aperture of the cylindrical wave coupled in the slab. A particularly interesting result from the shift selectivity is that it does not depend on the location $z$ of the hologram inside the slab and on the slab thickness $D$ but only on the wavelength of light and the N.A. of the spherical wave.

We performed experiments in a $\mathrm{LiNbO}_{3}$ crystal doped with $0.1 \% \mathrm{FeO}_{2}$. The crystal dimensions are $1 \mathrm{~mm} \times 20 \mathrm{~mm} \times 15 \mathrm{~mm}$, and it is cut for $90^{\circ}$ geometry. The reference beam is generated by focusing of light from a green cw laser (Coherent; wavelength, $0.532-\mu \mathrm{m}$, ordinary polarization) with a microscope objective on the 1-mm crystal's facet. The signal beam is a plane wave of diameter $7.5 \mathrm{~mm}$ propagating orthogonally to the $x, z$ plane as illustrated in Fig. 1(b). Figure 3(a) shows the selectivity curves for different locations $z_{i}(i=1,2,3)$ of the holograms along the slab. The shift selectivity is experimentally independent of the location $z_{i}$ as predicted from Eq. (5). With a N.A. of 0.18 outside the crystal and $\lambda=0.532 \mu \mathrm{m}$, Eq. (5) gives $\delta=1.5 \mu \mathrm{m}$, in agreement with the experimental value of $1.8 \mu \mathrm{m}$. Figure $3(\mathrm{~b})$ shows the results for a N.A. of 0.65. Again, shift selectivity is found to be independent of the location

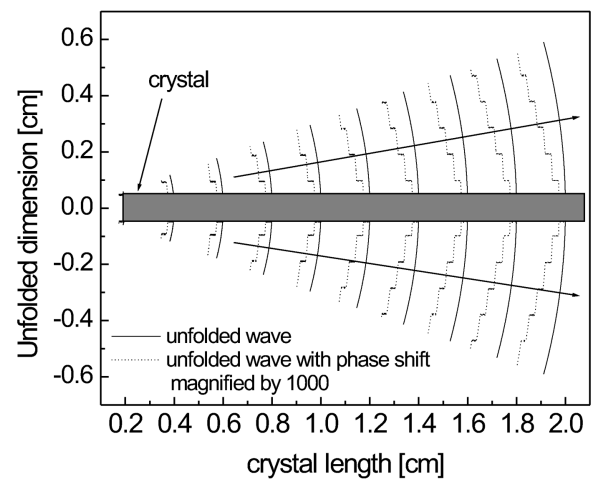

Fig. 2. Graphic representation of the unfolded cylindrical wave.
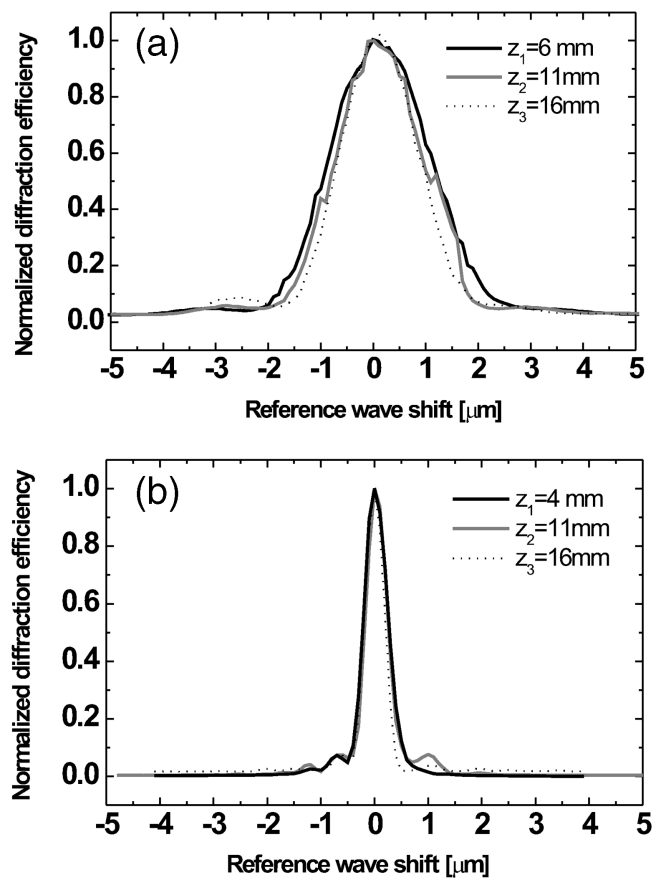

Fig. 3. Shift selectivity for different locations $z_{i} \quad(i=$ $1,2,3$ ) along the slab. (a) N.A. of the reference wave is 0.18 . (b) N.A. is 0.65 . 

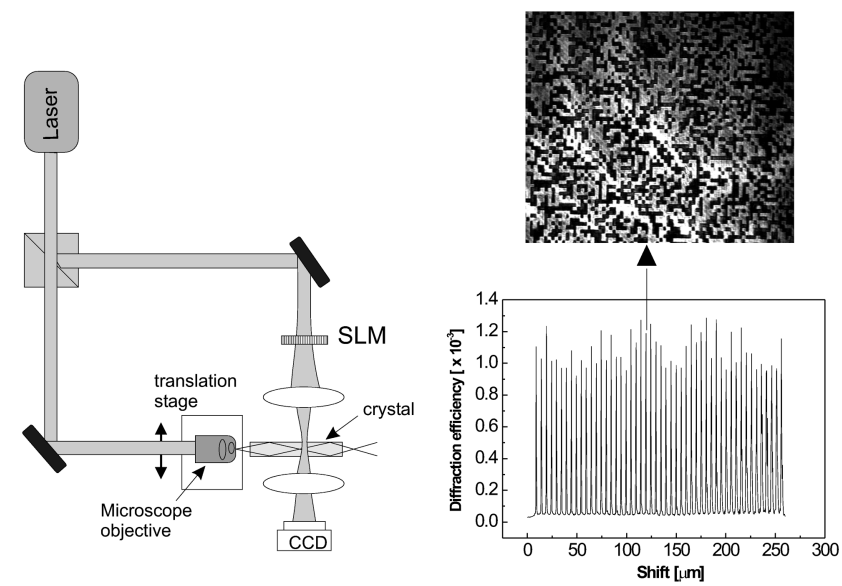

Fig. 4. (a) Setup for the 50 shift-multiplexed holograms. (b) Readout of 50 shift-multiplexed holograms in the slab crystal and sample reconstruction of hologram 20 .

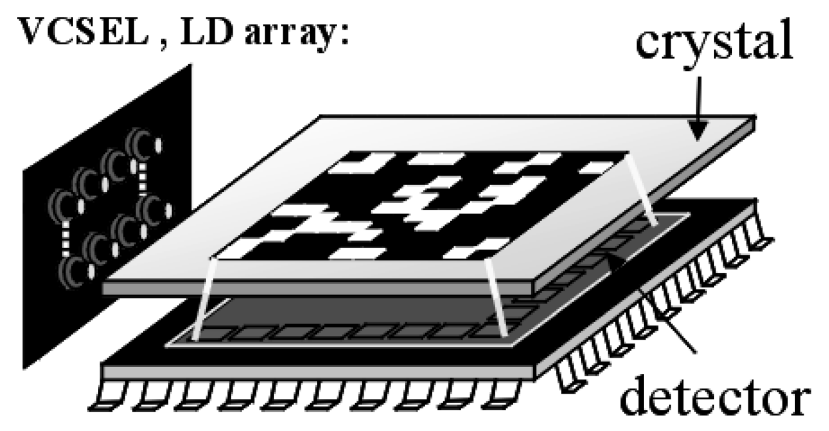

Fig. 5. Application of folded shift multiplexing to flat memory architectures. The natural N.A. of the vertical cavity surface emitting laser (VCSEL) array or the laser diode (LD) array is used as the spherical reference wave.

and slab thickness. Theoretical and experimental values agree very well $(0.41 \mu \mathrm{m}$ versus $0.5 \mu \mathrm{m})$.

By shifting the reference wave along the $y$ direction, we can store multiple hologram. We performed an experiment in which 50 images of random bit patterns were multiplexed by use of folded shift multiplexing. Figure 4 illustrates the experimental setup. An objective lens with a N.A. of 0.65 generates the spherical wave, which is coupled into the slab crystal. The spatial light modulator (SLM) is imaged with a 4- $f$ system onto a camera placed in close proximity to the slab. We record each hologram by shifting the spherical reference beam in the $y$ direction by $5 \mu \mathrm{m}$. The result of the experiment illustrated in Fig. 4 shows the diffraction efficiency versus the lateral shift of the reference beam in the $y$ direction. A holographic reconstruction of image 20 is also shown in the same figure. A signalto-noise ratio of 3.5 is measured for the reconstruction. The optical storage method presented here can lead to a very compact realization of holographic memories and holographic signal rerouting or reprogramming systems. Such a system is presented in Fig. 5.

We have presented an extension of shift multiplexing holographic storage by folding the spherical beam by use of total internal reflection in a holographic material slab. We showed that the folded shift selectivity in a $90^{\circ}$ geometry is independent of the slab thickness and in fact is equal to the standard shift selectivity. A shift selectivity of $0.5 \mu \mathrm{m}$ has been achieved with a 1-mm-thick material.

C. Moser's e-mail address is moser@ondax.com.

\section{References}

1. L. Hesselink and S. Redfield, Opt. Lett. 13, 877 (1988).

2. F. T. S. Yu, F. Zhao, H. Zhou, and S. Yin, Opt. Lett. 18, 1849 (1993).

3. K. H. Kim, H-S. Lee, and B. Lee, Opt. Lett. 23, 1224 (1998).

4. D. Psaltis, M. Levene, A. Pu, G. Barbastathis, and K. Curtis, Opt. Lett. 20, 782 (1995).

5. G. Barbastathis, M. Levene, and D. Psaltis, Appl. Opt. 35, 2403 (1996).

6. G. J. Steckman, A. Pu, and D. Psaltis, Appl. Opt. 40, 3387 (2001).

7. V. Markov, J. Millerd, and J. Trollinger, Opt. Lett. 24, 265 (1999).

8. S. Q. Tao, Z. Q. Jiang, and Q. Yuan, Chin. Phys. Lett. 17, 675 (2000).

9. A. Pu and D. Psaltis, Appl. Opt. 35, 2389 (1996).

10. L. Dhar, A. Hale, H. Katz, M. L. Schilling, M. Schnoes, and F. C. Schilling, Opt. Lett. 24, 487 (1999).

11. G. Steckman, V. Shelkovnikov, V. Berezhnaya, T. Gerasimova, I. Solomatine, and D. Psaltis, Opt. Lett. 25, 607 (2000).

12. B. E. A. Saleh and M. C. Teich, Fundamentals of Photonics (Wiley-Interscience, New York, 1991), p. 249. 